\title{
Bilateral 0.19 mg Fluocinolone Acetonide Intravitreal Implant in the Successful Treatment of Juvenile Idiopathic Arthritis-Associated Uveitis and Secondary Macular Oedema: A Case Report and Review of Intravitreal Therapies
}

\author{
Abdus Samad Ansari (D) - Zain Amir · Gwyn S. Williams
}

Received: November 22, 2020 / Accepted: December 16, 2020 / Published online: January 19, 2021

(c) The Author(s) 2021

\section{ABSTRACT}

Introduction: Treatment of juvenile idiopathic arthritis (JIA)-associated uveitis necessitates the use of long-term corticosteroids or immunosuppressive agents, each of which poses their own significant side effect profile. Initial treatment requires intensive topical glucocorticoids, with a step-up approach employing immunosuppressive agents for those cases with poor response or high-risk complications such as macular oedema. To date, there is minimal evidence to support a specific approach to such complicated subgroups. We present the first case to successfully employ the $0.19 \mathrm{mg}$ fluocinolone acetonide implant (ILUVIEN ${ }^{\circledR}$, Alimera Sciences, Hampshire, UK) as a novel device for prolonged intravitreal administration of disease-modifying agents for patients with JIA complicated by uveitis.

Methods: This retrospective case report describes a 20-year old woman diagnosed with oligoarticular JIA complicated by chronic uveitis and associated cystoid macular oedema (CMO). Considering factors including the patient's non-compliance, age, lens status, nonsteroid response, and good response to shortterm intravitreal steroid therapy, the $0.19 \mathrm{mg}$

\section{A. S. Ansari $(\bowtie) \cdot$ Z. Amir · G. S. Williams}

Singleton Eye Unit, Sketty Lane, Swansea SA2 8QA, UK

e-mail: samad.ansari@nhs.net fluocinolone acetonide intravitreal implant was deemed an appropriate step-up treatment option.

Results: At 12-month follow-up, the left eye (OS) showed an improvement in visual acuity to $6 / 15-1$ from $6 / 60+1$ (0.42 from 0.98 logMAR) (pre-insertion) and a reduction in central retinal thickness (CRT) of $199 \mu \mathrm{m}$ from $471 \mu \mathrm{m}$. The right eye (OD), treated 10 months later, showed an improvement in visual acuity to 6/7.5 from 6/24 - 1 (0.10 from 0.56 LogMAR) and a reduction in CRT of $327 \mu \mathrm{m} 6$ months after treatment.

Conclusion: In this case, the $0.19 \mathrm{mg}$ fluocinolone acetonide implant provided safe and effective long-term treatment of JIA-associated uveitis and secondary CMO. This potentially offers an alternative approach to complex cases that show good response to short-term corticosteroid use.

Keywords: Cystoid macular oedema; Fluocinolone acetonide; JIA; Uveitis 


\section{Key Summary Points}

There remains a significant paucity in literature regarding optimal treatment strategies for patients for whom systemic treatment may not be appropriate.

The use of short-term intravitreal implants has been confirmed to provide a safe and effective source of prolonged, steady intraocular delivery of corticosteroid.

Our case demonstrates the potential therapeutic benefits of the ILUVIEN ${ }^{\circledR}$ ( $0.19 \mathrm{mg}$ fluocinolone acetonide) in the treatment of JIA-associated uveitis.

Definitive safety and effectiveness can only be truly determined by larger clinical trials, which we believe are needed.

\section{DIGITAL FEATURES}

This article is published with digital features, including a summary slide, to facilitate understanding of the article. To view digital features for this article go to https://doi.org/10.6084/ m9.figshare.13387709.

\section{INTRODUCTION}

Uveitis poses significant long-term complications including permanent visual impairment [1]. Whilst the majority of paediatric cases are idiopathic in nature, infectious and autoimmune aetiologies are also implicated as potential disease sources. Of these conditions, juvenile idiopathic arthritis (JIA), carries the highest risk of morbidity due to its association with sight-threatening uveitis. JIA is the most prevalent rheumatic disease of childhood, with uveitis presenting as the most common extraarticular manifestation. With an estimated prevalence of about 113 cases per 100,000 , JIA is seen in close to $10 \%$ of these patients [1].
Treatment of JIA-associated uveitis often necessitates the use of long-term corticosteroids or immunosuppressive agents, each of which poses a significant side effect profile. Recent treatment algorithms published in the United Kingdom and Europe [1, 2] aim to ultimately achieve no activity in the anterior or posterior segments of the eye. Treatment is usually commenced on intensive topical glucocorticoids, with a step-up approach employing immunosuppressive agents for those cases with poor response or high-risk secondary features such as cystoid macular oedema (CMO). More recently, intravitreal implants containing $700 \mu \mathrm{g}$ of dexamethasone (sustained release) [3] and intravitreal injections of triamcinolone (used offlabel) [4] have been used in the treatment of JIA. However, given the chronic nature of the disease and the relatively short half-life of these forms of steroids, patients often require repeated injections-with their associated risks.

We present a case of bilateral JIA-associated uveitis with secondary macular oedema treated successfully with a $0.19 \mathrm{mg}$ fluocinolone acetonide intravitreal implant (ILUVIEN ${ }^{\circledR}$, Alimera Sciences, Hampshire, UK). To our knowledge, this is the first successful application of this therapy in this population. Description of this case provides clinicians with a potential alternative treatment approach for challenging cases of JIA-associated uveitis that are unresponsive to conventional therapy but show good response to short-term intravitreal corticosteroid therapy.

\section{CASE PRESENTATION}

A 20-year-old woman with a background of JIAassociated uveitis initially presented to our unit with a 2-month history of blurred vision and associated floaters. The patient's past medical history was significant for an oligoarticular form of JIA, diagnosed at age 8 whilst living in Eastern Europe. Her surgical history was significant for bilateral cataract surgery at 16 years of age. The aetiology of her cataracts was thought to be steroid-induced secondary to intensive topical treatment. Visual acuity was $6 / 36+1(0.82$ $\log$ MAR) in the right eye (OD) and 6/150 (1.39 
logMAR) in the left eye (OS). Examination identified anterior chamber inflammation: (OD) cells ++ , flare + , (OS) cells ++ , flare ++ and posterior vitritis $(\mathrm{OD})+,(\mathrm{OS})+++$. Given the nature of posterior uveitis and severity of disease, fundus examination was not possible; however, B-scans confirmed a flat retina and associated vitritis. Treatment was commenced consisting of topical prednisolone acetate 1\% and cyclopentolate $1 \%$. Blood tests on her initial attendance confirmed elevated P-ANCApositive titre and anti-nuclear antibody-positive titre. Her subsequent clinic appointment confirmed the previous diagnosis of JIA, with minimal response to topical therapy. She described joint issues since childhood, with a 5-year history of poorly managed complicated eye disease. Topical therapy provided improvement in vision [OD, 6/30 - 1 (0.68 logMAR); OS, 6/30 (0.70 $\log \mathrm{MAR})]$, but on the completion of optical coherence tomography (OCT) scans, $\mathrm{CMO}$ was identified on a background of chronic uveitis and central retinal thickness (CRT) (OS) of $471 \mu \mathrm{m}$. Alongside topical therapy, orbital floor triamcinolone was initially trialled in the worse affected eye (i.e., OS). Despite a good initial response, she presented within 2 months with a flare-up of uveitis. There remained bilateral chronic uveitis with macular oedema in both eyes (patient care illustrated in Fig. 3).

\section{COMPLIANCE WITH ETHICS GUIDELINES}

Written informed consent was obtained from the patient for the use of her medical records in writing this case report. The individual patient consent form is available on request. Formal ethics approval from the University Research Ethics Board was not required for the completion of this case report.

\section{FOLLOW-UP AND OUTCOMES}

Over the course of the next 12 months, a variety of topical corticosteroids, non-steroidal antiinflammatory drugs (NSAIDs), cycloplegics, and orbital floor triamcinolone treatment regimes were trialled. Discussions of systemic treatment took place; however, given a history of poor compliance and a potential risk of pregnancy, this was deemed inappropriate. Visual acuity during this period varied from 6/36 - $2(0.84$ $\log$ MAR) to $6 / 12-1$ (0.32 logMAR) (OD) and from 6/95 (1.12 logMAR) to counting fingers (OS). CRT ranged from 455 to $283 \mu \mathrm{m}$ (OD) and from 457 to $314 \mu \mathrm{m}$ (OS). Additionally, during this window, the patient's intraocular pressure (IOP) ranged between 12 and $21 \mathrm{mmHg}$ (OD) and 9 and $19 \mathrm{mmHg}$ (OS), with a vertical cupto-disc ratio (VCDR) of between 0.3 and 0.4 in both eyes. Given the patient was bilaterally pseudophakic and did not appear to be a steroid responder, an intravitreal form of sustained-release steroid implant was indicated. The intravitreal dexamethasone was initially trialled in the right eye and achieved improved visual acuity [6/7.5-1 (0.12 logMAR)] and a reduction in macular oedema. However, given this response, the patient was lost to follow-up. Unfortunately, after a series of non-attendances, 4 months after her last intravitreal therapy she became symptomatic once more, requiring further intervention. Due to the patient's non-compliance, age, lens status, nonsteroid response, and good response to intravitreal short-acting steroid therapy, the $0.19 \mathrm{mg}$ fluocinolone acetonide intravitreal implant (ILUVIEN $^{\circledR}$, Alimera Sciences, Hampshire, UK) was deemed to be a suitable treatment option.

In September 2018, the left eye was injected with a fluocinolone acetonide implant; the visual acuity was $6 / 60+1(0.98$ LogMAR) prior to treatment (Fig. 1). Follow-up confirmed complete resolution of $\mathrm{CMO}$ and quiet posterior segments in the ensuing 12 months. At this point the CRT in this left eye was $272 \mu \mathrm{m}$, with a concurrent improvement in visual acuity (Fig. 2), and the right eye was implanted 10 months after the left eye had been initially treated. Intraocular pressures post 


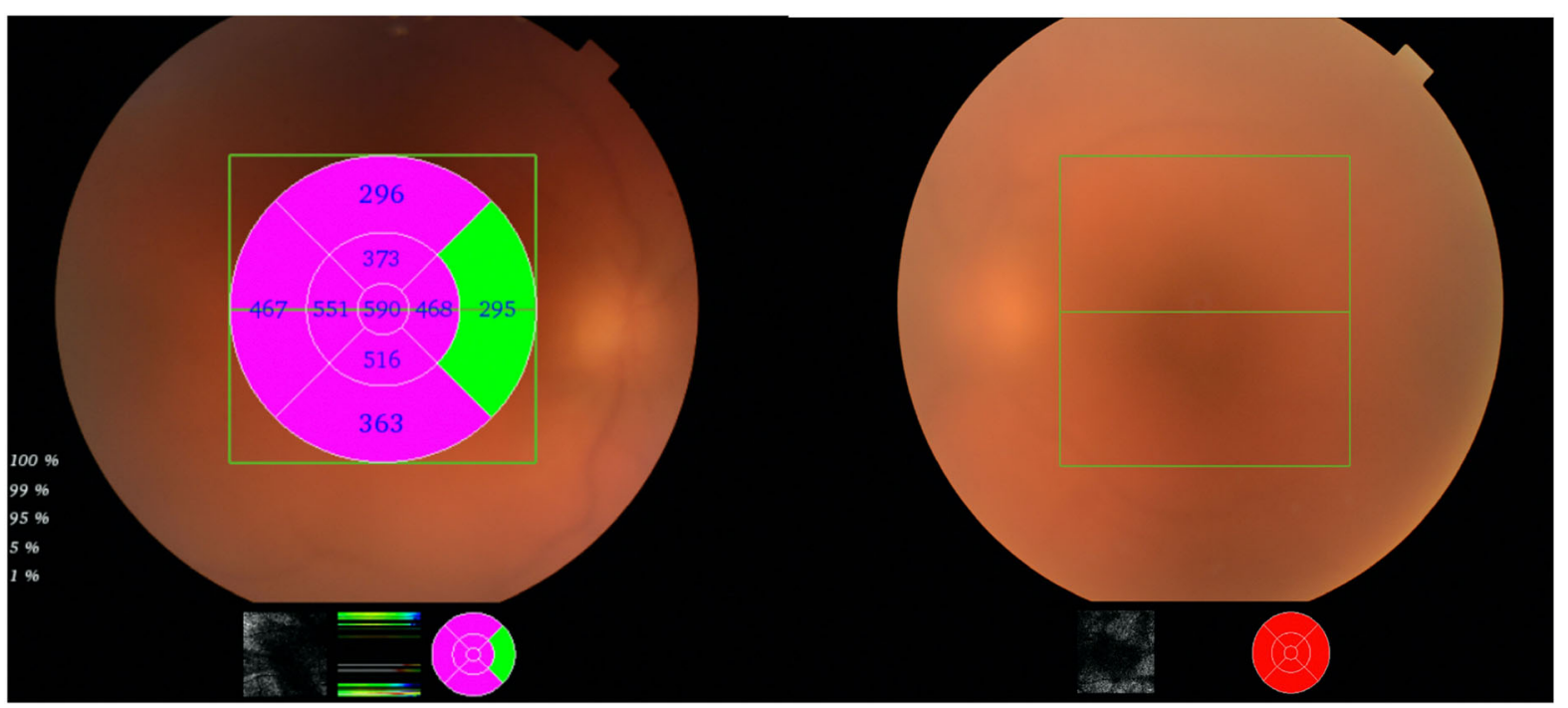

Fig. 1 Right and left eye prior to treatment. Exhibiting significant vitreous haze, unable to determine severity of cystoid macular oedema in left eye due to severity of vitritis

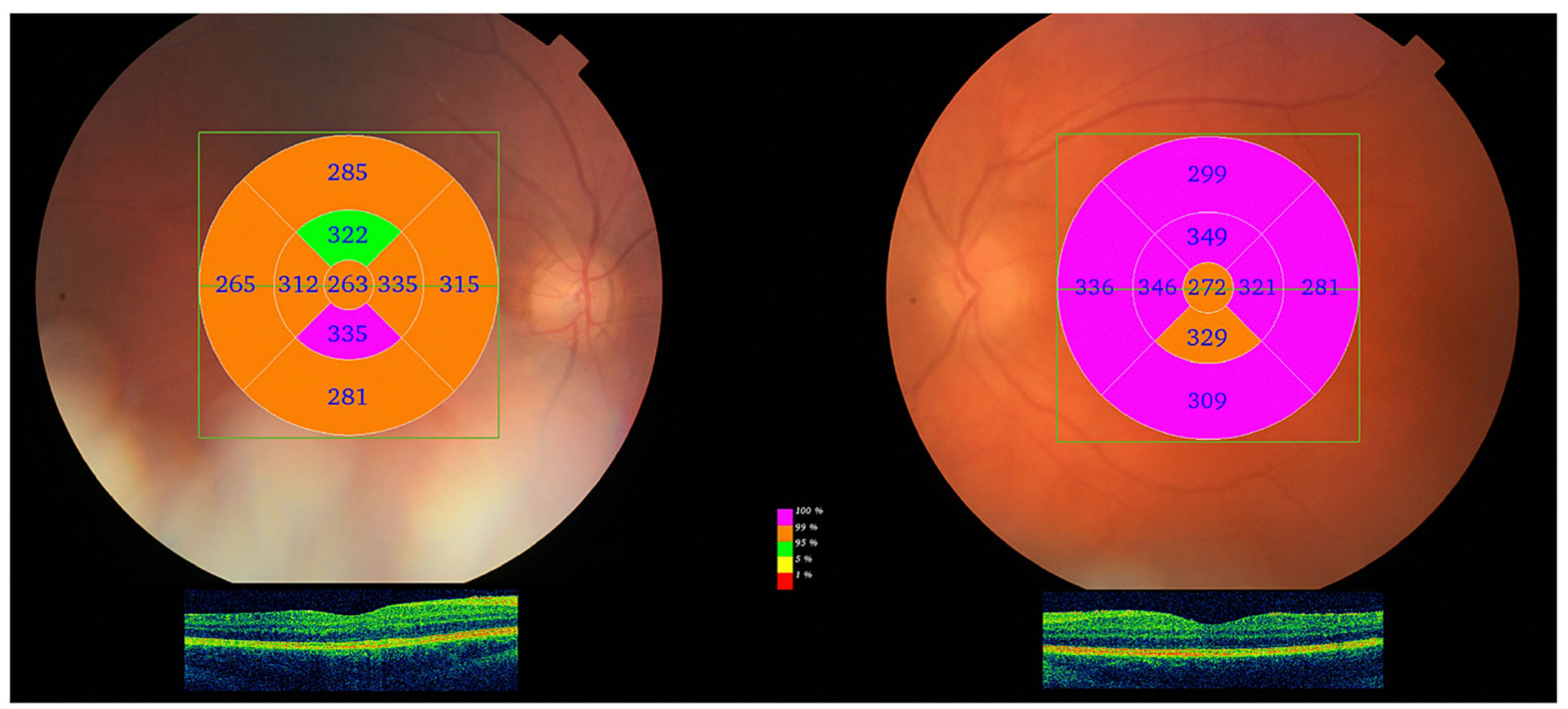

Fig. 2 Right eye 6 months after treatment and left eye 12 months after treatment. Exhibiting complete resolution of cystoid macular oedema and control of posterior segment inflammation

administration of the fluocinolone acetonide implant remained within below $21 \mathrm{mmHg}$ at all follow-up appointments. Visual acuity and CRT outcomes following implant can be seen in Table 1.

\section{DISCUSSION AND CONCLUSIONS}

Current treatment algorithms in the management of non-infectious uveitis of the posterior segment involve systemic, local, or topical preparations of corticosteroids. These agents can be used in conjunction with immunosuppressant therapies. Despite the success achieved employing these standard approaches to 
Table 1 Visual and retinal thickness outcomes

\begin{tabular}{|c|c|c|c|c|}
\hline & \multicolumn{2}{|l|}{ Visual acuity } & \multicolumn{2}{|c|}{$\begin{array}{l}\text { Central retinal } \\
\text { thickness }\end{array}$} \\
\hline & Right eye & Left eye & $\begin{array}{l}\text { Right } \\
\text { eye }\end{array}$ & $\begin{array}{l}\text { Left } \\
\text { eye }\end{array}$ \\
\hline Peak findings during care pre-implant & $6 / 36-2(0.84)$ & $\mathrm{CF}$ & 590 & 471 \\
\hline Pre-fluocinolone acetonide implant & $\begin{array}{l}6 / 24-1 \\
(0.56)\end{array}$ & $6 / 60+1(0.98)$ & 590 & * \\
\hline Post-fluocinolone acetonide implant ( 3 months) & $\begin{array}{l}6 / 7.5-1 \\
(0.12)\end{array}$ & $\begin{array}{l}6 / 18+2((0.52(0.4 \\
\mathrm{ph}))\end{array}$ & 272 & 280 \\
\hline $\begin{array}{l}\text { Post-fluocinolone acetonide implant (6 months RE and } \\
\text { LE) }\end{array}$ & $6 / 7.5(0.10)$ & $\begin{array}{l}6 / 30+1((0.72(0.42 \\
\mathrm{ph}))\end{array}$ & 263 & 299 \\
\hline Post-fluocinolone acetonide implant (12 months) & Awaiting & $6 / 15-1(0.42)$ & Awaiting & 272 \\
\hline
\end{tabular}

$C F$ count fingers, $P h$ pinhole, $N / A$ not applicable, $R E$ right eye, $L E$ left eye

*unable to quantify due to severity of vitritis

disease, effectiveness is often compromised when faced with intermediate and posterior forms of disease in subgroups of patients with high-risk complications. Thus, the management of JIA-associated uveitis requires an individualized approach based on the child's age, lens status, and tolerance to systemic treatment. Currently there remains a significant paucity in literature regarding optimal treatment strategies for patients for whom systemic treatment may not be appropriate. To our knowledge, the intravitreal administration of the fluocinolone acetonide implant for JIA has not been previously described. Our case highlights its successful application and proposes the need for further clinical studies evaluating its true safety, efficacy, and effectiveness in this patient cohort (Fig. 3).

To date, a number of corticosteroid-based implants have been reported to successfully treat non-infectious uveitis and stabilize disease activity. These include dexamethasone (OZURDEX $^{\circledR}$ ) and fluocinolone acetonide (ILUVIEN ${ }^{\circledR}$, RETISERT $\left.^{\circledR}\right)$. Unfortunately, there are limited data to address the effectiveness of such devices within distinct high-risk patients in whom disease is complicated by poor prognostic indicators, as observed in our patient with JIAassociated macular oedema.

The dexamethasone implant (OZURDEX) is a relatively short-acting implant with a duration of effect of between 4 and 6 months. Patients often require repeated injections given the disease course. It has been associated with a relatively high rate of post-administration raised intraocular pressure and cataract formation. The effect of repeated injections remains unknown; however, its efficacy has been proven in non-infectious intermediate or posterior uveitis [5]. Despite its known risk profile and the repeated injections required for optimal effect, OZURDEX has been shown to improve visual acuity and use of rescue medication, as demonstrated in the HURON study [5].

Conversely, the RETISERT implant requires surgical implantation and contains $-0.59 \mathrm{mg}$ of fluocinolone acetonide. It has been approved by the US Food and Drug Administration (FDA) but has no such license in the European Union [5]. Implantation of this device requires surgery, with the insert being placed through a pars plana incision and anchored to the sclera with sutures. Although there is a dramatically increased half-life in comparison to the dexamethasone implant, it has been associated 


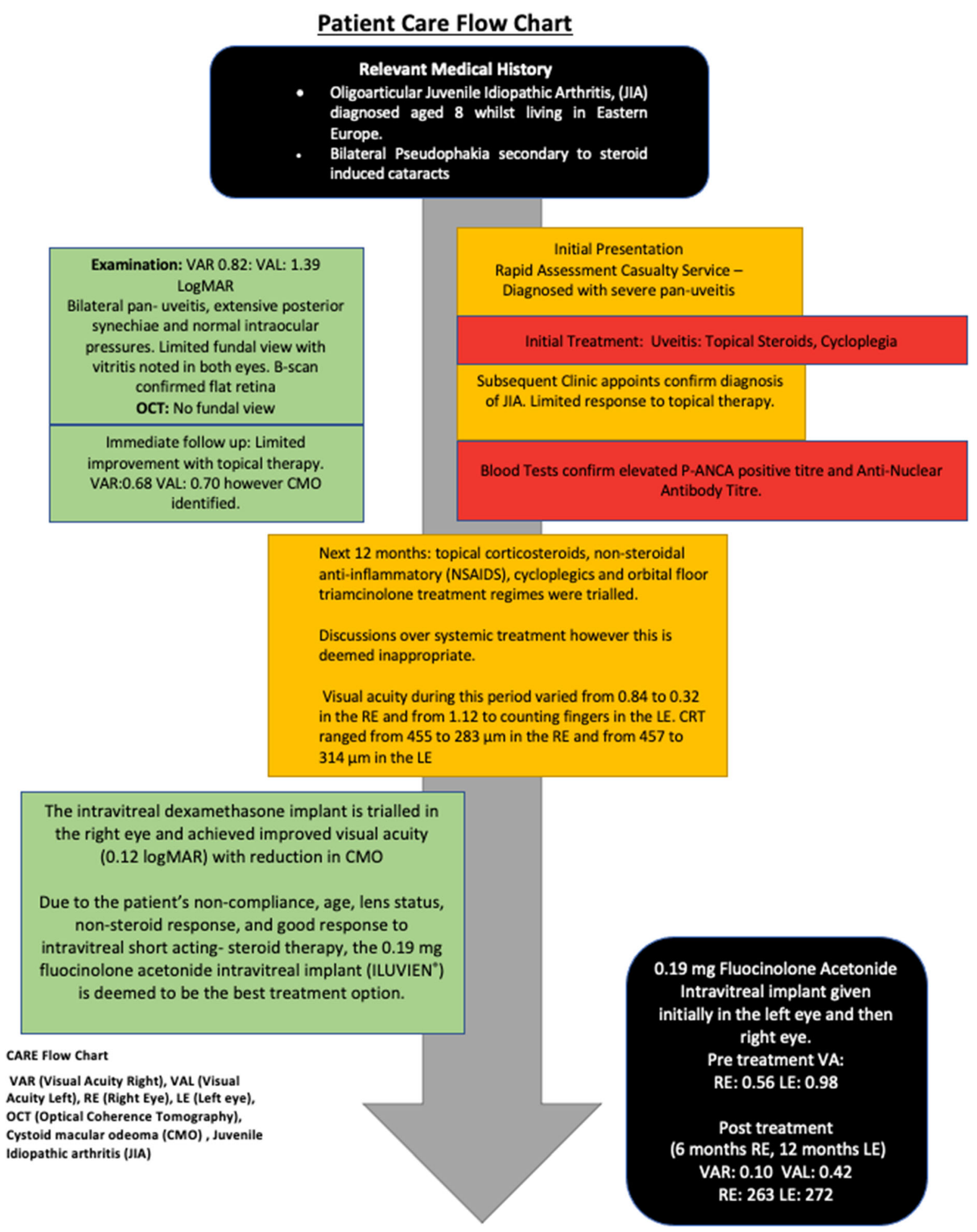

Fig. 3 Patient care flow chart. $V A R$ visual acuity right, $V A L$ visual acuity left, $R E$ right eye, $L E$ left eye, $O C T$ optical coherence tomography, $C M O$ cystoid macular oedema, $J I A$ juvenile idiopathic arthritis 
with a number of unwanted complications. Studies evaluating its efficacy have suggested post-insertion cataract rates of $93 \%$, and close to $51.1 \%$ of eyes requiring antihypertensive drops. One report evaluating its effectiveness primarily in JIA-associated uveitis determined that $50 \%$ of patients eventually required anti-glaucoma surgery [6], and of the eight patients followed up, one required retinal detachment surgery. Furthermore, all patients required anti-rheumatic drugs alongside the implant to treat the macular oedema [6].

The $0.19 \mathrm{mg}$ fluocinolone acetonide intravitreal implant (ILUVIEN, Alimera Sciences, Hampshire, UK) has the ability to release $0.2 \mu \mathrm{g} /$ day of fluocinolone acetonide for a period of up to 3 years. It is made of a non-biodegradable polymer measuring $3.5 \mathrm{~mm}$ in length and $0.37 \mathrm{~mm}$ in diameter, with its administration according to the same aseptic procedural norms required of any intravitreal injection. More recently its efficacy has been supported in the treatment of non-infectious uveitis inflammation and macular oedema, respectively [7-10]. A recently published multicentre randomized prospective sham-controlled study assessed its safety and efficacy in the management of inflammation in chronic non-infectious posterior uveitis. Findings at 6 and 12 months confirmed significantly reduced rates of recurrence and improved visual acuity, as well as reduced need for systemic and local adjunctive treatments [7]. Weber et al. conversely looked to evaluate its effectiveness in the treatment of noninfectious uveitic macular oedema. Although with a fairly limited sample size $(n=11)$, the study was able to achieve a median follow-up period of 19 months. Eighty-two percent of eyes presented with an improvement or inactive inflammation, with a mean CRT reduction of $168 \mu \mathrm{m}$ seen after treatment and no patients requiring antihypertensive drops or glaucoma surgery during the follow-up period.

Despite these positive reports, the generalizability of such interventions to high-risk subgroups of the JIA-associated uveitis population is questionable. Additional gaps in the literature remain, specifically the lack of long-term follow-up for known chronic remitting, relapsing diseases. Given the atypical course of disease, chronic uveitis in our patient consisted of recurrence, treatment, and quiescence before the cycle repeated. Limitations of such a procedure also include the lack of systemic control and risk of glaucoma. It is therefore vital that these patients are monitored closely by both ophthalmologist and rheumatologist, respectively. This should include regular assessment of the optic nerves to ensure that periodic pressure spikes are not missed. With each successive episode of inflammation there lies an inherent risk of incremental vision loss. Given the age, serial non-attendance, and poor compliance of our patient, it was essential that a sustained-released form of intravitreal therapy be administered.

In our case, the bilateral administration of the fluocinolone acetonide implant appeared to allow safe and effective long-term treatment of JIA-associated uveitis and secondary macular oedema. The patient continues to be regularly reviewed, given the implant maintains a slow release of up to 36 months, allowing continued monitoring of safety and benefit. Definitive efficacy and effectiveness can only be truly determined by larger clinical trials, which we believe are warranted.

\section{ACKNOWLEDGEMENTS}

We kindly thank the patient who generously consented for their medical history and clinical experience to be written up and repurposed for academic use. We would additionally like to thank Alimera Sciences Limited for their assistance in open access charges for this manuscript.

Funding. No external funding was required for the completion of this report. The journal's rapid service fee was provided by Alimera Sciences Limited, the manufacturer of Iluvien ${ }^{\circledR}$.

Additional Assistance. We would also like to thank Mr Chris Wright, Director of Scientific Communications, Alimera Sciences, for his assistance in reviewing our manuscript. No funding was used for this assistance. 
Authorship. All named authors meet the International Committee of Medical Journal Editors (ICMJE) criteria for authorship for this article, take responsibility for the integrity of the work as a whole, and have given their approval for this version to be published.

Authorship Contributions. GSW was the primary consultant caring for the patient. ASA completed the draft manuscript. ASA, ZA, and GSW critically appraised and revised the manuscript. Additionally, critical appraisal assistance was provided by Alimera Sciences Limited. All authors read and approved the final manuscript.

Disclosures. The authors Zain Amir and Gwyn S. Williams have nothing to disclose. Abdus Samad Ansari is now affiliated with Kings College Hospital NHS Trust, London, but has nothing else to disclose.

Compliance with Ethics Guidelines. Written informed consent was obtained from the patient for the use of their medical records in writing this case report. The individual patient consent form is available on request. Formal ethics approval from the University Research Ethics Board was not required for the completion of this case report.

Open Access. This article is licensed under a Creative Commons Attribution-NonCommercial 4.0 International License, which permits any non-commercial use, sharing, adaptation, distribution and reproduction in any medium or format, as long as you give appropriate credit to the original author(s) and the source, provide a link to the Creative Commons licence, and indicate if changes were made. The images or other third party material in this article are included in the article's Creative Commons licence, unless indicated otherwise in a credit line to the material. If material is not included in the article's Creative Commons licence and your intended use is not permitted by statutory regulation or exceeds the permitted use, you will need to obtain permission directly from the copyright holder. To view a copy of this licence, visit http:// creativecommons.org/licenses/by-nc/4.0/.

\section{REFERENCES}

1. Hawkins MJ, Dick AD, Lee RJ, et al. Managing juvenile idiopathic arthritis-associated uveitis. Surv Ophthalmol. 2016;61:197-210.

2. Bou R, Adán A, Borrás F, et al. Clinical management algorithm of uveitis associated with juvenile idiopathic arthritis: interdisciplinary panel consensus. Rheumatol Int. 2015;35:777-85.

3. Jinagal J, Gupta G, Agarwal A, et al. Safety and efficacy of dexamethasone implant along with phacoemulsification and intraocular lens implantation in children with juvenile idiopathic arthritis associated uveitis. Indian J Ophthalmol. 2019;67: 69.

4. Kok H, Lau C, Maycock N, McCluskey P, Lightman S. Outcome of intravitreal triamcinolone in uveitis. Ophthalmology. 2005;112:1916.e1911-1916.e1917.

5. Lowder C, Belfort R, Lightman S, et al. Dexamethasone intravitreal implant for noninfectious intermediate or posterior uveitis. Arch Ophthalmol. 2011;129:545-53.

6. Leinonen S, Immonen I, Kotaniemi K. Fluocinolone acetonide intravitreal implant $\left(\right.$ Retisert $\left.^{\circledR}\right)$ in the treatment of sight threatening macular oedema of juvenile idiopathic arthritis-related uveitis. Acta Ophthalmol. 2018;96:648-51.

7. Weber LF, Marx S, Auffarth GU, et al. Injectable 0. 19-mg fluocinolone acetonide intravitreal implant for the treatment of non-infectious uveitic macular edema. J Ophthalmic Inflam infect. 2019;9:3-18.

8. Mikhail M, Sallam A. Novel intraocular therapy in non-infectious uveitis of the posterior segment of the eye. Med Hyp Dis Innov Ophthalmol. 2013;2: 113.

9. Jaffe GJ, Foster CS, Pavesio CE, Paggiarino DA, Riedel GE. Effect of an injectable fluocinolone acetonide insert on recurrence rates in chronic noninfectious uveitis affecting the posterior segment: twelve-month results. Ophthalmology. 2019;126:601-10.

10. Jaffe GJ, Pavesio CE, Study Investigators. Effect of a fluocinolone acetonide insert on recurrence rates in noninfectious intermediate, posterior, or panuveitis: three-year results. Ophthalmology. 2020;127(10):1395-404. 